2008-03

Regulation of multiple angiogenic pathways by DII4 and Notch in human umbilical vein endothelial cells.

\title{
Harrington, LS
}

http://hdl.handle.net/10026.1/10301

\subsection{6/j.mvr.2007.06.006}

Microvasc Res

All content in PEARL is protected by copyright law. Author manuscripts are made available in accordance with publisher policies. Please cite only the published version using the details provided on the item record or document. In the absence of an open licence (e.g. Creative Commons), permissions for further reuse of content should be sought from the publisher or author. 


\title{
Regulation of multiple angiogenic pathways by Dll4 and Notch in human umbilical vein endothelial cells
}

\author{
Laura S. Harrington $^{\mathrm{a}}$, Richard C.A. Sainson ${ }^{\mathrm{a}}$, Cassin Kimmel Williams ${ }^{\mathrm{a}}$, Jennifer M. Taylor ${ }^{\mathrm{b}}$, \\ Wen Shi ${ }^{\text {a }}$, Ji-Liang $\mathrm{Li}^{\mathrm{a}}$, Adrian L. Harris ${ }^{\mathrm{a}, *}$ \\ ${ }^{a}$ Cancer Research UK, Molecular Oncology Laboratories, Weatherall Institute of Molecular Medicine, John Radcliffe Hospital, \\ University of Oxford, Oxford, OX3 9DS, UK \\ ${ }^{\mathrm{b}}$ Wellcome Trust Centre for Human Genetics, University of Oxford, Roosevelt Drive, Oxford, OX3 7BN, UK
}

Received 19 February 2007; revised 15 June 2007; accepted 15 June 2007

Available online 29 June 2007

\begin{abstract}
The Notch ligand, D114, is essential for angiogenesis during embryonic vascular development and is involved in tumour angiogenesis. Several recent publications demonstrated that blockade of D114 signalling inhibits tumour growth, suggesting that it may constitute a good candidate for anti-cancer therapy. In order to understand the role of D114 at the cellular level, we performed an analysis of Dll4-regulated genes in HUVECs. The genes identified included several angiogenic signalling pathways, such as VEGF, FGF and HGF. In particular we identified downregulation (VEGFR2, placenta growth factor PlGF) of VEGF pathway components resulting in the overall effect of limiting the response of HUVEC to VEGF. However extensive upregulation of VEGFR1 was observed allowing continued response to its ligand PlGF but the soluble form of the VEGFR1, sVEGFR1 was also upregulated. PIGF enhanced tubulogenesis of HUVEC suggesting that downregulation of P1GF and upregulation of VEGFR1 including sVEGFR1 are important mechanisms by which Dl14 attenuates P1GF and VEGF signalling. Dl14stimulated HUVECs had impaired ERK activation in response to VEGF and HGF indicating that Dll4 signalling negatively regulates these pathways. Dll4 expression reduced vessel sprout length in a 3D tubulogenesis assay confirming that Dll4 signalling inhibits angiogenesis. Altogether, our data suggest that D114 expression acts as a switch from the proliferative phase of angiogenesis to the maturation and stabilisation phase by blocking endothelial cell proliferation and allowing induction of a more mature, differentiated phenotype. The regulation of sVEGFR1 provides a novel mechanism for Dll4 signalling to regulate cells at distance, not just in adjacent cells.
\end{abstract}

(C) 2007 Elsevier Inc. All rights reserved.

Keywords: D114; Notch; cDNA microarray; HUVEC; Sprout formation; Angiogenesis

\section{Introduction}

Notch signalling has recently been implicated in vascular development and homeostasis since genes encoding components of Notch signalling are mutated in two human diseases (Alagille Syndrome and CADASIL (Cerebral Autosomal Dominant Arteriopathy with Subcortical Infarcts and Leukoencephalopathy)) which exhibit vascular defects (Karsan, 2005; Shawber and Kitajewski, 2004). Furthermore, many Notch receptors and ligands are expressed in cells of the vasculature

\footnotetext{
* Corresponding author. Fax: +44 1865222431.

E-mail address: aharris.lab@cancer.org.uk (A.L. Harris)
}

(Iso et al., 2003a; Villa et al., 2001). Finally studies of transgenic mice have revealed an essential early role for Notch signalling in angiogenesis (Iso et al., 2003a).

Notch signalling is an evolutionarily conserved intercellular signalling pathway mediated by membrane-tethered receptorligand interactions between adjacent cells (Artavanis-Tsakonas et al., 1999; Lai, 2004). Receptor-ligand binding induces sequential cleavages of the Notch receptor, the last of which is performed by the $\gamma$-secretase complex, releasing the Notch intracellular domain (NICD) which translocates to the nucleus (Artavanis-Tsakonas et al., 1999; Lai, 2004). In the nucleus

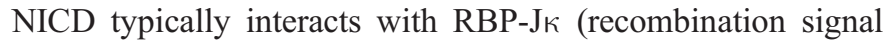
binding protein $\mathrm{J}_{\kappa}$ ) leading to the transcription of Notch target genes such as members of the Hes and Hey families of 
transcriptional repressors (Iso et al., 2003b). In mammals there are four Notch receptors (Notch1-4) and 5 Notch ligands: two serrate-like ligands named Jagged1 and Jagged2 and three delta-like ligands (D1l) named Dll1, 3, 4.

We and others have shown that D114 is expressed specifically at sites of vascular development and angiogenesis and is confined to arterial ECs (Benedito and Duarte, 2005; Claxton and Fruttiger, 2004; Mailhos et al., 2001; Shutter et al., 2000). D114 expression is particularly critical for angiogenesis as haploinsufficiency of Dl14 leads to embryonic lethality in mice due to vascular defects (Duarte et al., 2004; Gale et al., 2004; Krebs et al., 2004) demonstrating the essential role of Dll4 in angiogenesis during development.

Thus Dl14 expression is essential for normal angiogenesis during development but D114 expression in adults is confined to areas of physiological angiogenesis such as in the ovary around developing follicles and areas of pathological angiogenesis such as tumour vasculature (Mailhos et al., 2001). Recent evidence suggests that Dll4 expression is upregulated in tumour endothelium and that this correlates with tumour vessel maturation and remodeling (Hainaud et al., 2006; Patel et al., 2006). In addition Dll4 expression is induced by hypoxia, a common feature of tumour development known to induce angiogenesis (Mailhos et al., 2001) making Dll4 an attractive anti-tumour target. Indeed recent papers have demonstrated that blockade of Dll4 signalling promotes non-productive angiogenesis thus inhibiting tumour growth (Noguera-Troise et al., 2006; Ridgway et al., 2006) though the mechanistic basis for this remains unclear. In the present study we performed a cDNA microarray screen to identify Dll4 regulated genes in order to understand the function of D1l4 signalling in physiological and pathological angiogenesis.

\section{Materials and methods}

\section{Cell culture, cells and reagents}

HUVECs were isolated from fresh human umbilical cords by infusion with $0.2 \%$ collagenase. Single donor HUVECs were used between passages 3 and 7 and were cultured in M199 media supplemented with $20 \%$ fetal calf serum (FCS: Sigma-Aldrich, St. Louis, MO), $12 \mathrm{mM}$ L-glutamine, $50 \mathrm{mg} / \mathrm{L}$ ECGS (endothelial cell growth supplement; BD Biosciences Bedford MA, USA), 10 units/mL heparin (Sigma) and an antimycotic/antibiotic (Gibco). The Phoenix amphotropic viral packaging cell line (gift from Garry Nolan) was cultured in DMEM supplemented with $10 \%$ FCS, $12 \mathrm{mM}$ L-glutamine and penicillin/streptomycin. Recombinant human Dl14 extracellular domain (rDll4) was purchased from R\&D Systems (Minneapolis, USA). The $\gamma$-secretase inhibitor DAPT (Calbiochem) was dissolved in DMSO (Sigma) and used at a final concentration of $2 \mu \mathrm{M}$.

\section{Retroviral packaging and infection}

The retroviral vector and full length human D114 construct have been previously described (Williams et al., 2006). Low passage, 50\% confluent HUVECs were infected using $0.4-\mu \mathrm{m}$ filtered virus containing supernatant supplemented with $4 \mathrm{ng} / \mathrm{mL}$ polybrene. After $5 \mathrm{~h}$ at $37^{\circ} \mathrm{C}$ an equal volume of normal HUVEC culture media was added. Infection efficiencies were from $60 \%$ to $90 \%$.

\section{Microarray analysis}

Microarray analysis was performed in triplicate and each replicate used HUVECs from a different donor. Total RNA was extracted from HUVECs using TRI-reagent (Sigma) followed by DNase I treatment (DNase-free; Ambion) according to the manufacturer's instructions. First and second strand cDNA synthesis was performed using Superscript dscDNA Synthesis Kit (Invitrogen) and $10 \mu \mathrm{g}$ of total RNA. Clean-up of double stranded cDNA was carried out using Phase Lock Gels, $2 \mathrm{~mL}$ light (Eppendorf), followed by synthesis of labeled cRNA with the BioArray High Yield RNA Transcript Labeling Kit (ENZO, Affymetrix). Purification of cRNA and quantification was done with RNeasy Mini Kit (Qiagen), followed by cRNA Fragmentation using $30 \mu \mathrm{g} c$ RNA and Fragmentation Buffer (200 mM Tris-acetate pH 8.1, 500 mM MKOAc, $150 \mathrm{mM}$ $\mathrm{MgOAc})$. Hybridisation Cocktail for human HG-U133A Affymetrix GeneChip ${ }^{\circledR}$ utilised the Gene Chip Eurkaryotic Hybridisation Control Kit (ENZO, Affymetrix) and hybridisation, washing, staining, and scanning of the chip was performed according to the manufacturer's instructions. Data was labeled as MIAME compliant. Raw signal files were background corrected and normalised using the gcrma modification of the rma normalisation procedure (Irizarry et al., 2003) available from the Bioconductor project (www.bioconductor.org) for the R statistical language. $\log 2$ ratios of D114 signal to control signal were generated for each probeset. A list was generated that contained those probesets for which an average absolute fold change of at least 1.5 was observed between Dll4 and control samples. Where a gene was represented by more than one probeset the average signal value was used. This list was used to generate a list of statistically significant $(P \leq 0.01)$ probesets with the eBayes approach (Smyth, 2004) as implemented in the limma package (Smyth and Speed, 2003) of BioConductor. Visualisation of this gene list was performed using the hierarchical clustering algorithm with euclidean distance and single linkage. Enrichment analysis was conducted using the DAVID algorithm and significance of enrichment is measured using the Fisher's Exact Test for each GO term relative to the background of the Affymetrix HG-U133A chip.

\section{Quantitative real time PCR (Q-PCR)}

Reverse transcription was performed using $1 \mu \mathrm{g}$ total DNase 1-treated RNA and the High Capacity cDNA Archive Kit (Applied Biosystems). The resulting cDNA was used for Q-PCR using the Exiqon system (Roche, Basel, Switzerland). Briefly Q-PCR reactions were set up in triplicate using the Corbett Research Roto Gene RG-3000 robot (Corbett, Sydney, Australia). Each $25 \mu 1$ reaction contained the equivalent of $25 \mathrm{ng}$ reverse transcribed cDNA, $0.4 \mu \mathrm{M}$ of each oligonucleotide, $12.5 \mu 1$ of $2 \times$ Absolute QPCR master mix (AbGene, Epsom, UK) and $0.25 \mu 1$ of the appropriate Exiqon probe. GAPDH or Flo2 were used as reference genes to normalise results. The cycling conditions used were: $95^{\circ} \mathrm{C}$ for $10 \mathrm{~min}$ followed by 40 cycles of $95^{\circ} \mathrm{C}$ for $15 \mathrm{~s}$ and $60^{\circ} \mathrm{C}$ for $60 \mathrm{~s}$. The relative quantitation was performed as previously described (Patel et al., 2005). The differences between Q-PCR experimental groups were analyzed using one sample Student's $t$ test and $p<0.05$ was considered significant.

\section{Oligonucleotides}

\begin{tabular}{lll}
\hline Gene & Forward 5'-3' & Reverse 5'-3' \\
\hline GAPDH & AGCCACATCGCTCAGACAC & GCCCAATACGACCAAATCC \\
Flo2 & CTCAGCTTCACCATCAAGGAC & TCAGCATCTCTCTGCACCAC \\
Sulfatase1 & CCAATGCTTCCCAACACATA & GCATTGGTCCTGTGTACTGC \\
Elastin & CACTGGGGTATCCCATCAAG & GTGGTGTAGGGCAGTCCATAG \\
PCDH12 & GGAGGAGCTCACTGTGGATT & CTGATGCAGCAAGGACAGC \\
INHBA & CTCGGAGATCATCACGTTTG & CCTTGGAAATCTCGAAGTGC \\
\hline
\end{tabular}




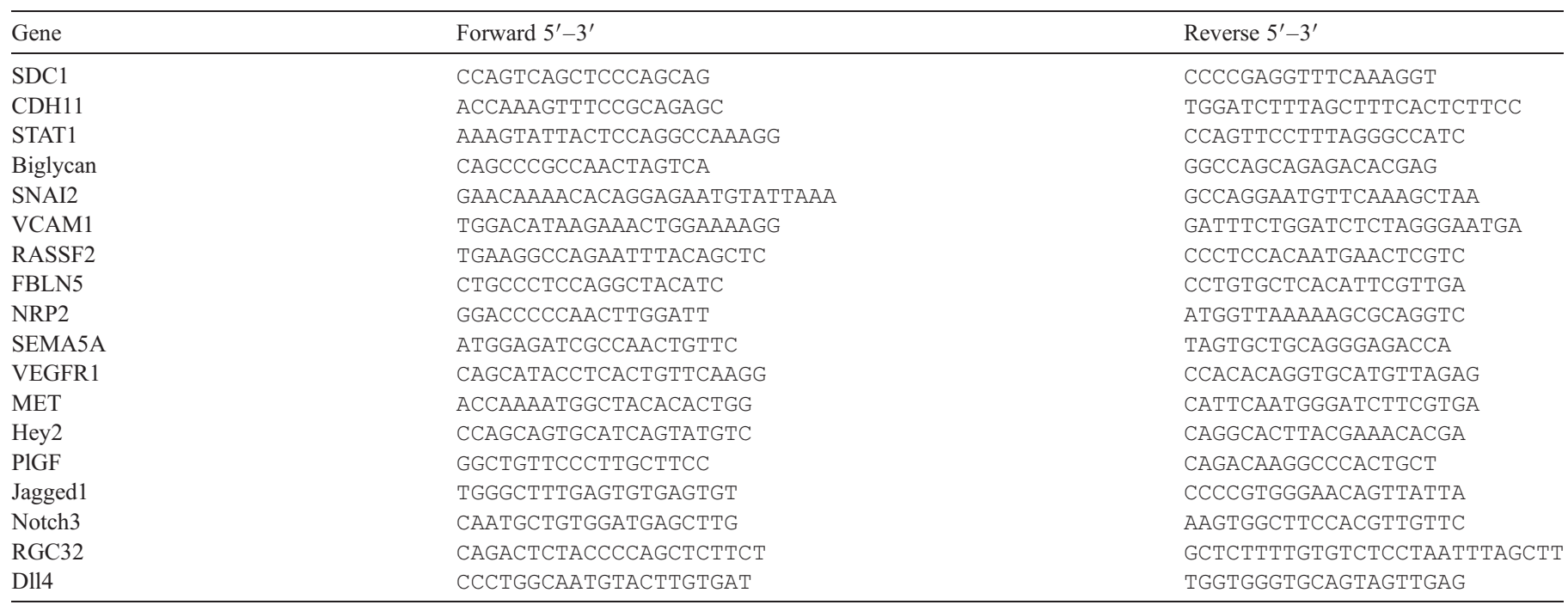

\section{rDll4-coated tissue culture plates}

Tissue culture plates were coated with $0.2 \%$ gelatine $(\mathrm{w} / \mathrm{v})$ in $\mathrm{PBS}$ containing $1 \mu \mathrm{g} / \mathrm{mL}$ rDll4 or BSA as a control (Williams et al., 2006) and incubated at $4{ }^{\circ} \mathrm{C}$ for $24 \mathrm{~h}$ before use. Plates were warmed to $37{ }^{\circ} \mathrm{C}$ and the coating solution aspirated prior to seeding $2 \times 10^{5}$ HUVECs per well in 6-well plates. RNA, protein (as per serum stimulation of HUVECs below) or conditioned media were harvested $48 \mathrm{~h}$ later.

\section{$\gamma$-Secretase treatment}

$2 \times 10^{5}$ HUVECs were seeded in the presence of $2 \mu \mathrm{M}$ DAPT or an equivalent amount of DMSO vehicle. The media containing DAPT or DMSO control was replaced after $24 \mathrm{~h}$ (except when used for ELISA). RNA, protein (as per stimulation of HUVECs below) or conditioned media was harvested after $48 \mathrm{~h}$.

\section{Western blotting}

Proteins were separated by SDS-PAGE using standard techniques. Antibodies were purchased from the following companies: Notch1IC, MET, STAT1, phospho-ERK and total ERK antibodies (Cell Signalling), Jagged1, Notch3, VEGFR2, Snai2 (Santa Cruz), $\beta$-tubulin, $\beta$-actin, VEGFR1 antibodies (Sigma). The anti-Hes1 antibody was a gift from Tetsuo Sudo, Toray Industries, Japan. Anti-mouse and anti-rabbit HRP conjugated secondary antibodies were from DAKO while anti-goat HRP was from Perbio.

\section{$V G_{165}, H G F$ and PlGF stimulation of HUVECs}

$2 \times 10^{5}$ HUVECs per well were seeded into BSA control or rDll4-coated 6well plates. After $43 \mathrm{~h}$ the media was replaced with starvation media (DMEM with $0.5 \% \mathrm{FCS}$ ). $5 \mathrm{~h}$ later the media was replaced with starvation media (unstimulated) or starvation media supplemented with $20 \mathrm{ng} / \mathrm{mL} \mathrm{VEGF}_{165}$ or HGF for 2 or $10 \mathrm{~min}$ or $10 \mathrm{ng} / \mathrm{mL}$ PlGF for $2.5,5$ and $10 \mathrm{~min}$. Cells were lysed in RIPA buffer (Sigma) supplemented with Complete protease inhibitor cocktail (Roche) and phosphatase inhibitor cocktails I and II (Sigma). Following centrifugation $\left(13,000 \mathrm{rpm}, 5 \mathrm{~min}\right.$ at $\left.4{ }^{\circ} \mathrm{C}\right)$ the supernatant was collected. Equal amounts of protein were separated by SDS-PAGE as described above.

In vitro 3D-tubulogenesis fibrin gel bead assay

The fibrin gel bead assay was previously described (Nakatsu et al., 2003). HUVECs were infected with retrovirus encoding for GFP (empty vector control) or full-length Dl14 as described above and subsequently placed in the fibrin gel bead assay. To investigate the effect of PIGF, HUVEC were placed in the fibrin gel bead assay and treated 2 days later with vehicle- or $10 \mathrm{ng} / \mathrm{mL}$ or $50 \mathrm{ng} / \mathrm{mL}$ PlGF-containing EGM-2, replacing the media every other day for a total of 5 treatments. A Zeiss Axiovert S100 microscope using 10x and 20x objectives attached to a Hamamatsu C4742-95 camera was used to capture images. Image acquisition software used was OpenLab 3.5 and Image J software (NIH) was used for the analysis of sprout length (Nakatsu et al., 2003).

\section{Soluble VEGFR1 ELISA}

$2 \times 10^{5}$ HUVEC were seeded on BSA or rDll4-coated plates as described above except that EGM-2 media was used. ELISA for human SVEGFR1 (R\&D Systems) was performed according to the manufacturer's instructions using the conditioned media (diluted 1 in 10 in EGM-2 media) from HUVECs cultured on BSA- or rDll4-coated plates in the presence of DMSO or DAPT for $48 \mathrm{~h}$. Total ng of sVEGFR1 were normalised to the amount of cellular protein in $\mathrm{mg}$ to account for differences in proliferation.

\section{Results}

\section{Exogenous Dll4 enhances Notch signalling in HUVECs}

HUVECs were chosen for this study because of their capacity to express low levels of D114 mRNA as detected by RTPCR (Patel et al., 2005) and to induce D114 expression following hypoxia and VEGF stimulation (Hainaud et al., 2006; Liu et al., 2003; Mailhos et al., 2001; Patel et al., 2005). Transduction of HUVECs with Dll4-encoding retrovirus increased D114 expression at the mRNA and protein levels as previously reported (Williams et al., 2006).

\section{Identification of Dll4 target genes by microarray analysis}

In order to identify genes and processes regulated by Dll4 signalling that are responsible for its role in regulating angiogenesis we performed cDNA microarray analysis in triplicate comparing Dll4-retrovirally-infected HUVECs to control empty vector-infected HUVECs. Analysis of the microarray data revealed 104 candidate genes that were statistically significantly up or downregulated by an average 


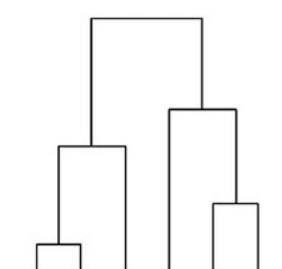

Gene Symbol Fold Change
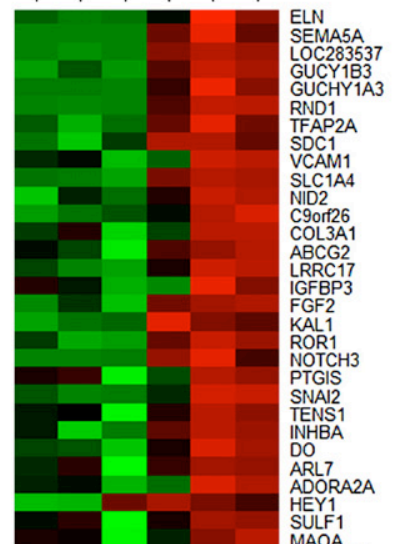
GUCY1B3 RND1 TFAP2 2 SDC1
VCAM1
SLC1A4 SLC1A4 Colf26 ABCG IGFBP3 KAL1 ROR1 NOTCH3 PTGIS TENS1
INHBA
DO
ARL7 ARL7
ADORA2A
HEY1 SULF1 MAOA ADAMTS
PDLIM3 PDLIM3
AMPH LTBP4 4 PLSCR
PLVAP
STAT1 STAT1 EFNB2 MEST
FABP4 FABP4
RAPGEF5
CDH11 ILIR1 SETBP1 BDNF
ATP 10D SLIT2
LMCD1
PHTF2 PHTF2
JAG1
HES1
SAT NR2F1 BAMBI FLT1 TOX SART2
SLIT3
SULT1B1 ATXN1 ATP 11B GPR126
SULT1A3 CRSP2 CRSP2
KCTD12 TPM1
PCDH12 SVIL SH3BP5 JUN RGL1
SLC38A1 SLC38A ITGA6 ADM
MPP1
P2RX4 NAV 3 CHST7
KRT19
ORP10 ORP10
SOX17 MT2 MAFF
MT1X C1QR1 NRP1 IGFBP2 GALNT6 DYSF PHLDA RASSF2 PDE4B
PRKAR2B MMP 10 RGC32 of more than 1.5 fold in Dll4-infected HUVECs compared to control-infected HUVECs $(p<0.01$; Fig. 1 and Supplementary Table 1). Table 1 shows that Dll4 regulated a wide variety of genes including components of cell signalling pathways important for angiogenesis such as the VEGF, FGF and hepatocyte growth factor (HGF) signalling pathways. Indeed the list of 104 genes is significantly enriched for GO terms of angiogenesis $(p=0.00012)$, and angiogenesis-associated processes, including proliferation $(p=0.018)$ and cell adhesion $(p=0.029)$. The gene functional classification algorithm within DAVID produced a cluster of angiogenesis related GO terms that had a geometric mean significance of enrichment of $(p=0.0012)$. Dll4 upregulated more genes than it downregulated.

\section{Validation of candidate Dll4 target genes by Q-PCR}

Using Q-PCR we analysed the mRNA levels of candidate genes in Dll4-infected HUVECs compared to empty vectorinfected HUVECs for a panel of 14 genes up-regulated in the microarray analysis and 5 genes that were down regulated. Genes were chosen from the list of 104 genes and also a longer list of genes regulated by Dll4 by 1.5 fold or more (Supplementary Table 2). Q-PCR results for the 19 genes tested confirmed the microarray data (Table 1). As expected using HUVECs from different donors the magnitude of gene expression changes by Q-PCR was not always similar but the average fold induction or repression of genes by Dll4 was at least 1.5 fold matching that of the microarray.

\section{Dll4 target genes are regulated by immobilised Dll4}

In order to confirm the regulation of genes by Dll4 signalling by an alternative non-viral approach, HUVECs were cultured on dishes coated with recombinant human D114 extracellular domain (rD114) or with BSA as a control. Immobilisation of the Notch ligand is thought to mimic the tethering of the ligand on the cell surface enabling Notch signalling which soluble ligands usually fail to do as has been shown for Deltal (Varnum-Finney et al., 2000). This approach activated Notch signalling as demonstrated by the accumulation of the cleaved active N1IC in HUVECs cultured on rDll4 for $48 \mathrm{~h}$ (Fig. 2B).

Q-PCR validation of a subset of the candidate genes by this method showed that all 12 genes tested were up-regulated and all 5 were downregulated in HUVECs cultured on rDll4 compared to those cultured on BSA-coated dishes (Table 1). This was in complete agreement with the cDNA microarray and Q-PCR results from retroviral-infected HUVECs. In addition this suggests that the regulation of these genes is via Notch receptor cleavage rather than via Dl14-ICD as the recombinant protein lacks the ICD.

Fig. 1. A heat map to show genes differentially regulated by D114 expression in HUVECs. The heatmap and dendrogram show clustering of samples based on the $\log 2$ expression ratio of 104 probesets. Clustering is based on manhattan distance metric and single linkage. Heatmap colours represent $\log 2$ ratio of -5 (green) to 5 (red). 
Table 1

Genes involved in angiogenesis-related signalling pathways and biological processes identified as D1l4 target genes by microarray analysis and Q-PCR validation

\begin{tabular}{llll}
\hline Genes (UniGene number) & $\begin{array}{l}\text { Microarray } \\
(n=3)\end{array}$ & Infection & $\begin{array}{l}\text { rDll4-coated } \\
\text { plates }\end{array}$
\end{tabular}

\begin{tabular}{llll}
\hline $\begin{array}{l}\text { Signalling pathways } \\
\text { VEGF }\end{array}$ & & \\
NRP1 (Hs.131704) & -3.9 & ND & ND \\
NRP2 (Hs.471200) & -2.7 & $-1.9[0.2](3)$ & $-1.6[0.1](3)$ \\
P1GF (Hs.252820) & -6.9 & $-3.3[0.6](4)$ & $-2.8[0.6](4)$ \\
VEGFR1 (Hs.507621) & 2.8 & $2.6[0.4](3)$ & $3.9[0.5](3)$ \\
VEGFR2 (Hs.479756) & -6.3 & ND & ND \\
FGF & & & \\
FGF2 (Hs.284244) & 7.3 & ND & ND \\
HGF & & & $-2.3[0.1](3)$ \\
MET (Hs.132966) & -1.9 & $-1.9[0.3](3)$ & \\
Notch & & & $3.1[0.2](3)$ \\
Notch 3 (Hs.8546) & 7.0 & $9.7[4.2](5)$ & $12.9[0.3](3)$ \\
Hey2 ${ }^{\S}$ (Hs.14427) & 1.2 & $14.8[6.0](3)$ & $-2.2[0.4](3)$ \\
RASSF2 (Hs.379970) & -6.8 & $-2.1[0.4](4)$ & \\
Jagged1 (Hs.224012) & 3.2 & $1.5[0.1](5)$ & $2.6[0.3](3)$
\end{tabular}

\section{Proliferation}

Fibulin5 (Hs.332708)

Inhibin $\beta A$ (Hs.28792)

STAT1 (Hs.470943)

$\begin{array}{lll}3.0 & 5.8[1.4](3) & 3.4[0.3](3) \\ 5.6 & 5.0[0.7](5) & 8.1[0.9](4) \\ 4.3 & 1.8[0.1](5) & \text { ND }\end{array}$

Migration and adhesion

CDH11 (Hs.116471)

CXCR4 (Hs.421986)

PCDH12 (Hs.439474)

Snai2 (Hs.360174)

VCAM1 (Hs.109225)

$\begin{array}{rll}3.9 & 2.3[0.2](5) & \mathrm{ND} \\ -10.4 & \mathrm{ND} & \mathrm{ND} \\ -1.9 & -6.2[1.3](5) & -7.0[1.0](5) \\ 6.6 & 3.9[0.5](5) & 4.9[1.2](4) \\ 12.5 & 15.0[4.7](4) & 8.8[2.3](4)\end{array}$

Vessel guidance

RND1 (Hs.124940)

Sema5A (Hs.321637)

Slit2 (Hs.558436)

Slit3 (Hs. 145601)

$\begin{array}{rll}14.5 & \text { ND } & \text { ND } \\ 28.9 & 22.6[6.8](3) & 15.1[3.9](3) \\ 3.4 & \text { ND } & \text { ND } \\ 2.8 & \text { ND } & \text { ND }\end{array}$

Differentiation and morphogenesis

\begin{tabular}{|c|c|c|c|}
\hline Elastin (Hs.252418) & 30.1 & $143[49](5)$ & $60.0[33.6](4)$ \\
\hline EphrinB2 (Hs.149239) & 4.2 & ND & ND \\
\hline Integrin $\beta 3$ (Hs.218040) & -1.6 & ND & ND \\
\hline \multicolumn{4}{|c|}{ Extracellular matrix-associated } \\
\hline Biglycan (Hs.821) & 2.3 & $5.5[1.3](5)$ & $6.7[1.0](4)$ \\
\hline $\begin{array}{l}\text { Collagen, typeIII, alpha } 1 \\
\quad(\text { Hs.443625) }\end{array}$ & 8.4 & ND & ND \\
\hline $\begin{array}{l}\text { Matrix metalloprotease } \\
10 \text { (Hs.2258) }\end{array}$ & 9.8 & ND & ND \\
\hline SDC1 (Hs.224607) & 13.0 & $8.5[2.2](5)$ & $6.0[1.2](4)$ \\
\hline Sulfatase1 (Hs.409602) & 5.4 & $23.3[8.2](5)$ & $8.8[4.1](4)$ \\
\hline
\end{tabular}

Genes identified as D114 targets by cDNA microarray analysis categorized according to the signaling pathway or biological process in which they play a role. The average fold change in mRNA levels according to the microarray analysis is shown. In addition the table shows a comparison between the average fold change in mRNA levels of 20 genes identified by microarray analysis to be differentially regulated by D114 signalling either in Dll4-expressing HUVECs compared to empty vector control-infected HUVECs (infection) or HUVECs cultured on Dll4coated plates compared to HUVECs cultured on BSA-coated plates as a control (rDll4-coated plates). The average fold change relative to expression in the corresponding control cells is shown. ND, not determined. Parentheses, number of independent experiments performed on HUVECs from separate donors. Square brackets, S.E.M. All results are significant with $p<0.05 .{ }^{\S}$ While Hey 2 mRNA was not regulated by more than 1.5 fold in the microarray, Q-PCR results demonstrate that it is upregulated by D114 signalling using both approaches.
Dll4 signalling regulates Hes1, Jagged1, MET, VEGFR1, Snai2 and STAT1 proteins

In order to determine whether Dll4-induced mRNA changes were reflected at the protein level we performed western blotting for some of the confirmed targets and found that levels of Hes 1 protein were higher in HUVECs expressing Dll4 by retroviral infection and in HUVECs cultured on rDll4-coated plates compared to control (Fig. 2A). Since the Q-PCR validation by both approaches to induce Dll4 signalling yielded similar results as did the western blotting for Hes1 (Fig. 2A) we then tested the protein expression of STAT1, Jagged1, VEGFR1, snai2 and MET in HUVECs cultured on rDll4-coated plates compared to control. In HUVECs cultured on rDll4-coated plates, Notch signalling was activated and this correlated with higher levels of STAT1, Jagged1, Snai2 and full length VEGFR1 proteins and a lower level of MET protein compared to control (Fig. 2B). These protein expression changes correlated with the mRNA level changes induced by Dll4.

In addition we used the $\gamma$-secretase inhibitor DAPT to prevent Notch signalling and investigated its effect on Dll4induced regulation of elastin (the most upregulated gene by QPCR), PCDH12 (the most down-regulated gene by Q-PCR) and two other candidates. DAPT treatment inhibited the Dll4induced upregulation of elastin, sulfatase-1 and snai2 and downregulation of PCDH12 at the mRNA level (Fig. 2C). DAPT treatment inhibited Dll4 activation of Notch signalling as demonstrated by the reduced accumulation of NICD in HUVECs cultured on Dll4-coated plates in the presence of DAPT (Fig. 2D). Furthermore DAPT treatment reduced the protein expression changes of VEGFR1, VEGFR2, MET, snai2 and STAT1 induced in HUVECs cultured on rDll4-coated plates (Fig. 2D).

\section{Dll4-Notch signalling regulates the levels of soluble VEGFRI}

Since D114 signalling upregulated the levels of full length VEGFR1 protein in HUVEC, the effect of Dll4 signalling on the levels of the inhibitory soluble alternative splice variant of VEGFR1 (sVEGFR1) in the conditioned media of HUVEC cultured on BSA or rDll4-coated plates was measured by ELISA. The levels of sVEGFR1 were elevated in the conditioned media of HUVEC cultured on Dll4-coated plates compared to those cultured on BSA by 3.5 fold (Fig. 2E). Treatment with DAPT inhibited the Dll4-induced elevation of sVEGFR1 confirming that Notch signalling is necessary for rDl14 to upregulate sVEGFR1.

\section{ERK activation in response to VEGF 165 and $H G F$ is impaired by Dll4 signalling}

Since Dll4 signalling altered the gene expression of several genes involved in VEGF signal transduction (Fig. 2; Table 1) we explored the effect of Dll4 signalling on the ability of HUVECs to respond to $\mathrm{VEGF}_{165}$ stimulation. We investigated the phosphorylation of ERK as ERK lies downstream of VEGFR activation. HUVECs cultured on 
rD114-coated plates for $48 \mathrm{~h}$ prior to starvation and stimulation with $20 \mathrm{ng} / \mathrm{mL} \mathrm{VEGF}_{165}$, had an impaired ability to phosphorylate and therefore activate ERK (Fig. 3). In addition, in a similar experiment, ERK activation in response to $20 \mathrm{ng} / \mathrm{mL}$ HGF stimulation was also impaired demonstrating that the decreased level of MET protein following Dll4 stimulation reduced the ability of HUVEC to respond to HGF (Fig. 3).

\section{Dll4 effects on sprout length in a 3D-tubulogenesis assay}

We investigated the effect of Dll4 expression in a 3Dtubulogenesis assay that recapitulates crucial steps characteristic of in vivo angiogenesis such as vessel elongation, vessel branching and lumen formation (Nakatsu et al., 2003). In the present study, expression of Dll4 clearly inhibited sprout formation (Fig. 4A). Indeed, HUVECs infected with D1l4encoding retrovirus only formed short vessels (Fig. 4A) which were about $50 \%$ of the length of mock or GFP-encoding empty vector control-infected HUVECs (Fig. 4B). These experiments demonstrated that increased Dll4-induced Notch signalling attenuated tubulogenesis.

\section{PlGF enhances tubulogenesis}

In order to investigate whether modulation of VEGF signalling pathway components in the tubulogenesis assay may contribute to the Dll4-induced phenotype described above, we examined the effect of PlGF in this assay. As shown in Fig. 5A, PlGF treatment led to enhanced tubulogenesis. PlGF is downregulated by Dll4 signalling, which has the opposite effect on tubulogenesis in this assay (Fig. 4), suggesting that downregulation of PlGF by Dll4 may contribute to Dll4-induced attenuation of tubulogenesis.

\section{Dll4 signalling enhances PlGF-induced ERK activation}

Since Dll4 signalling increased levels of the full-length VEGFR1 receptor the ability of PlGF, the ligand for VEGFR1, to activate ERK in HUVECs cultured on Dll4-coated plates compared to BSA-coated plates was investigated. PlGFstimulated activation of ERK was enhanced in HUVEC cultured on rDll4-coated plates and was also more prolonged (Fig. 5B).

\section{Discussion}

D114, the most recently identified Notch ligand, is essential for angiogenesis during embryonic vascular development. Like VEGF, haploinsufficiency of Dll4 is embryonic lethal, demonstrating its importance for successful angiogenesis (Duarte et al., 2004; Gale et al., 2004; Krebs et al., 2004). Recent reports have shown that blockade of D1l4 decreases tumour growth but increases vessel density in contrast to the previously accepted dogma that increased vessel density is associated with increased tumour growth (Noguera-Troise et al., 2006; Ridgway et al., 2006). However the underlying mechanisms by which Dll4-mediated Notch signalling contributes to the control of multiple signalling pathways to coordinate such complex vascular processes remains unclear. We have recently shown that Dll4 signalling increases tumour growth and decreases vessel density but the resulting vessels are larger and better perfused ( $\mathrm{Li}$ et al., submitted). We therefore suggest that D114 signalling may bring to an end the initial proliferative phase of angiogenesis thus accounting for the decreased vessel number and trigger a maturation phase to refine and stabilise vessels to improve vascular function. This idea is supported by recent in vivo data in Dll4+/- mice and using pharmacological inhibitors of Dll4-Notch signalling which demonstrate enhanced angiogenic sprouting and increased vascular proliferation when Dll4 levels or signalling is reduced in the retina or whole embryos (Hellstrom et al., 2007; Lobov et al., 2007; Scehnet et al., 2007; Suchting et al., 2007). However it is unclear how Dll4 regulates these processes and investigating downstream mechanisms was the purpose of this study. Here we identify Dll4-regulated genes which may constitute the mechanism by which Dll4 signalling coordinates such events.

The validity of using a microarray screen to identify Dll4 target genes is demonstrated by the fact that we have identified previously known Notch target genes such as Hey1, and Hes1 (Iso et al., 2003b) consistent with other reports (Patel et al., 2005; Williams et al., 2006).

Dll4 is highly expressed in tumour endothelium and is induced by VEGF and hypoxia (Hainaud et al., 2006; Liu et al., 2003; Mailhos et al., 2001; Patel et al., 2005). Dll4 signalling is known to decrease levels of VEGFR2 and the VEGF co-receptor NRP1 (Williams et al., 2006) but here we demonstrate that D114 signalling regulates several more components of VEGF signalling including NRP2 and PlGF (downregulated) and VEGFR1 (upregulated). These findings support recent in vivo data where VEGFR2 expression was elevated and VEGFR1 expression was decreased in the retina of Dll4+/- mice compared to wild type controls (Suchting et al., 2007).

By sequestration of VEGF away from VEGFR2 through higher receptor-ligand affinity (Ferrara et al., 2003), VEGFR1 can act as a negative regulator of VEGF action by inhibiting proliferation. Furthermore we have shown here that the inhibitory soluble splice variant of VEGFR1, sVEGFR1, is also upregulated in response to Dll4 contributing to the impairment of VEGF signalling. PIGF can stimulate angiogenesis possibly by displacing VEGF from VEGFR1 (Ferrara et al., 2003). Indeed we have shown enhanced tubulogenesis induced by PlGF in the 3D tubulogenesis assay in agreement with a previous report of PlGF-induced tubulogenesis using primary microvascular endothelial cells (Cai et al., 2003). Here we also show that the increased levels of full length VEGFR1 receptor following Dll4 signalling can enhance ERK activation in response to PlGF thereby indicating that the down regulation of PlGF by Dll4 is an important part of the programme of gene expression changes of VEGF pathway components induced by Dll4 resulting in impaired VEGF signalling. The inhibition of tubulogenesis by D1l4 in the 3D 
A.

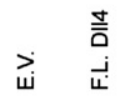$$
\text { 帋 咅 }
$$

Hes-1

$\beta$-Actin

c.

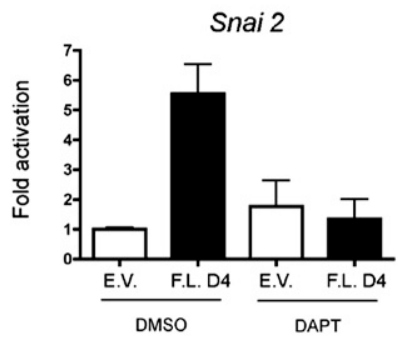

Sulfatase-1

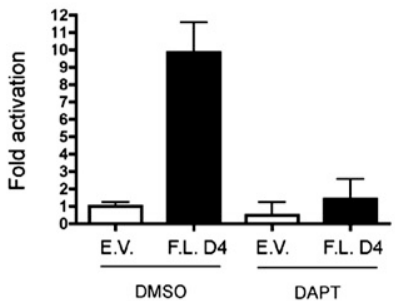

Elastin
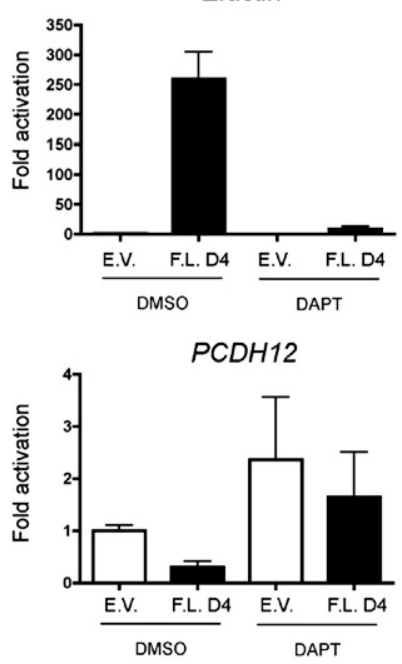

B. 飠 咅

N1ICD

STAT1

Jagged-1

VEGFR1

Snai2

MET

Actin

D.

$\frac{\text { DMSO }}{\text { BSA DLL4 }} \frac{\text { DAPT }}{\text { BSA DLL4 }}$
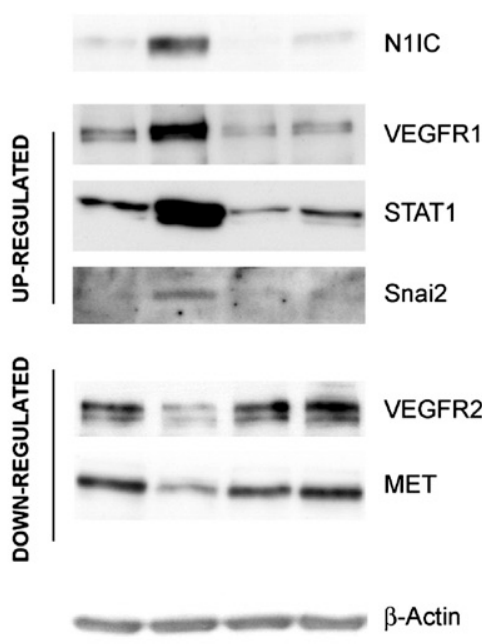

E.

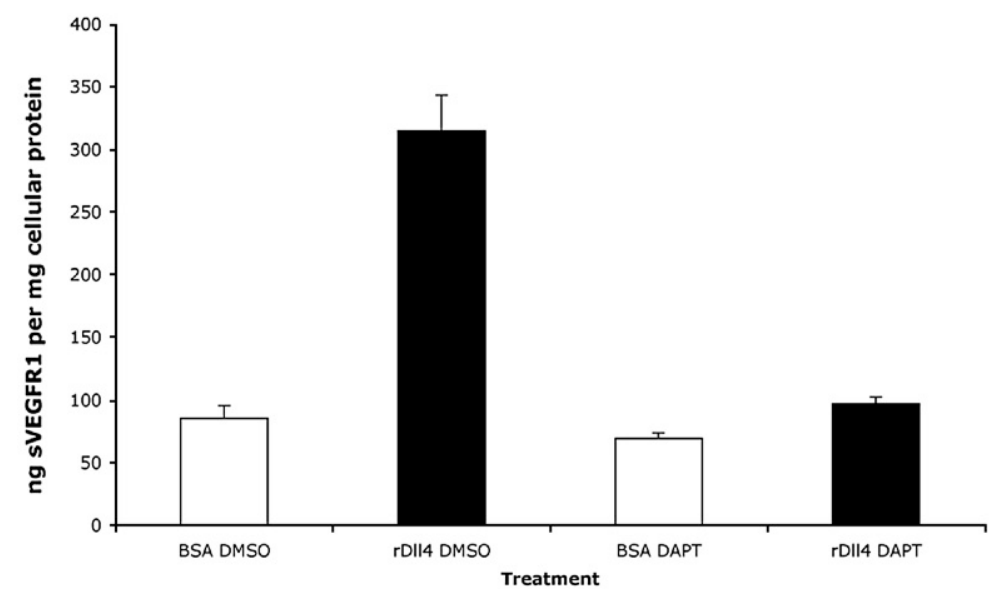




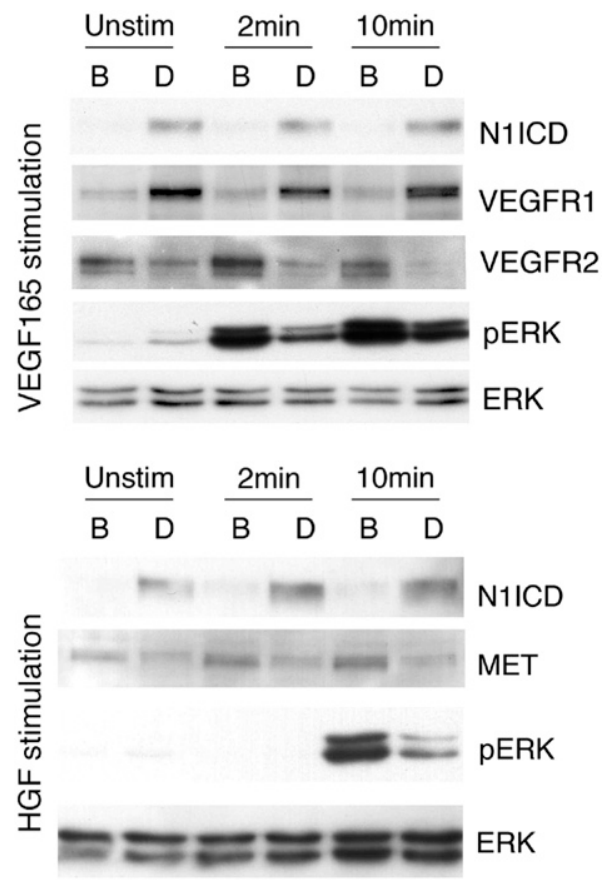

Fig. 3. Activation of ERK by $\mathrm{VEGF}_{165}$ and HGF stimulation is impaired in HUVECs cultured on rDll4-coated plates. HUVECs were cultured on Dll4coated plates for $48 \mathrm{~h} .5 \mathrm{~h}$ prior to harvesting the cells were starved and then left unstimulated or stimulated with the indicated growth factor $(20 \mathrm{ng} / \mathrm{mL})$ for $2 \mathrm{~min}$ or $10 \mathrm{~min}$. Activation of ERK is indicated by detection of phosphorylated ERK p42/44 with a phospho-specific antibody and was attenuated in HUVECs cultured on rDll4-coated plates. The blot was stripped and reprobed with total ERK as a loading control. One experiment representative of two is shown.

tubulogenesis assay (Fig. 4) is opposite to the effect of PlGF in the same assay suggesting that downregulation of PIGF contributes to this Dll4 phenotype.

Thus these data describe an efficient negative feedback loop whereby Dll4 is induced by VEGF but then reduces the ability of EC to respond to VEGF by regulating multiple components of the VEGF pathway. We demonstrate that all these changes result in impaired activation of ERK in response to $\mathrm{VEGF}_{165}$ stimulation in HUVEC cultured on Dll4-coated plates. That this impairment of ERK signalling might contribute to the reduced sprout length in the 3D-tubulogenesis assay is supported by the observation that inhibition of ERK activation reduced tube formation in a similar collagen gel tubulogenesis assay (Yang et al., 2004).
The upregulation of sVEGFR1 by Dl14-Notch signalling is particularly important as its levels could be monitored in serum making it a potentially useful diagnostic tool to identify patients who may benefit from anti-Dll4 therapy and to monitor their response to such therapy. In addition, regulation of the expression of sVEGFR1 by Dll4 raises the possibility that rather than only influencing angiogenesis via signalling to adjacent cells, Dl14 may have an impact on angiogenesis over greater distances by regulation of such soluble factors. Although exogenous PIGF showed some enhancement of signalling associated with full length VEGFR1 upregulation, this was not as extensive as the upregulation of VEGFR1, and it is possible that secreted sVEGFR1 limited this response, although fresh medium was used to stimulate the cells.

Notch signalling is known to inhibit ECs proliferation (Noseda et al., 2004; Sainson et al., 2005; Williams et al., 2006). Results from the cDNA microarray indicate further mechanisms by which D114 may limit EC proliferation for example by upregulating the levels of fibulin5 and STAT1 which are known to be involved in reducing EC proliferation (Albig et al., 2006; Battle et al., 2006). A reduction in EC proliferation could contribute to the reduced sprout length phenotype in the $3 \mathrm{D}$ tubulogenesis assay and lead to the reduced vessel number in Dll4-expressing tumours in vivo (Noguera-Troise et al., 2006; Li et al., submitted).

Activation of the HGF receptor, MET, induces cell proliferation, migration and tubulogenesis and branching morphogenesis and HGF regulates angiogenesis in vivo (Rosario and Birchmeier, 2003). A constitutively active form of the Notch4 receptor has been shown to inhibit MET expression in MDAMB-435ß4 cells (Stella et al., 2005) but here we demonstrate that Dll4 signalling downregulated MET mRNA and protein. Furthermore we showed that this Dll4-induced reduction of MET protein impairs the ability of HUVEC to respond to HGF stimulation and activate ERK. These results suggest that Dl14 signalling can negatively modulate key angiogenic pathways.

Dll4 is specifically expressed in arterial ECs (Benedito and Duarte, 2005; Claxton and Fruttiger, 2004; Mailhos et al., 2001) leading to the hypothesis that it is involved in the differentiation of ECs as they mature and that tumour vasculature is arterial in nature. Our data showed that Dll4 signalling downregulated the venous-specific gene NRP2 and upregulated genes associated with arterial vessels such as ephrinB2 (Iso et al., 2006) and elastin which is necessary for the elasticity of arterial vessels. Such a hypothesis is

Fig. 2. Gene expression changes induced by Dll4 are reflected at the protein level and are inhibited by the $\gamma$-secretase inhibitor DAPT. (A) Protein extracts from infected HUVECs or HUVECs cultured on BSA- or Dll4-coated plates for $48 \mathrm{~h}$ were separated by SDS-PAGE and immunoblotted. The levels of Hes1 protein (30 kDa) were increased following Dll4 stimulation by both approaches. (B) Culture of HUVECs on Dll4-coated plates compared to BSA-coated plates for $48 \mathrm{~h}$ led to an upregulation of STAT1 (84/91 kDa), Jagged1 (150 kDa), full length VEGFR1 (220 kDa), snai2 (30 kDa) and a downregulation of MET protein (145 kDa). (C) Control empty vector retroviral infected cells (E.V.) or full length Dll4-retroviral infected HUVECs (F.L.D4) were cultured for 2 days in the presence of $2 \mu M$ DAPT or the equivalent amount of DMSO as a control. DAPT prevented the Dll4-induced mRNA expression changes of elastin, Snai2, sulfatase-1 and PCDH12. One experiment representative of three is shown. mRNA expression changes are represented as a fold change relative to expression in E.V. cells in DMSO. (D) HUVECs were cultured on BSA or rDll4-coated plates for $48 \mathrm{~h}$ in the presence of $2 \mu \mathrm{M}$ DAPT or the equivalent amount of DMSO. DAPT prevented the D114-induced changes in protein levels of VEGFR1, VEGFR2, STAT1, MET and Snai2. Blots representative of three independent experiments are shown. (E) HUVECs were cultured on BSA or rDll4-coated plates for $48 \mathrm{~h}$ in the presence of $2 \mu \mathrm{M}$ DAPT or the equivalent amount of DMSO. HUVECs cultured on rDll4-coated plates for $48 \mathrm{~h}$ secreted 3.5 -fold more sVEGFR1 into the conditioned media than the control HUVEC per mg cellular protein and this was inhibited by DAPT treatment. Error bars represent standard deviation. One experiment representative of two is shown. 
A.
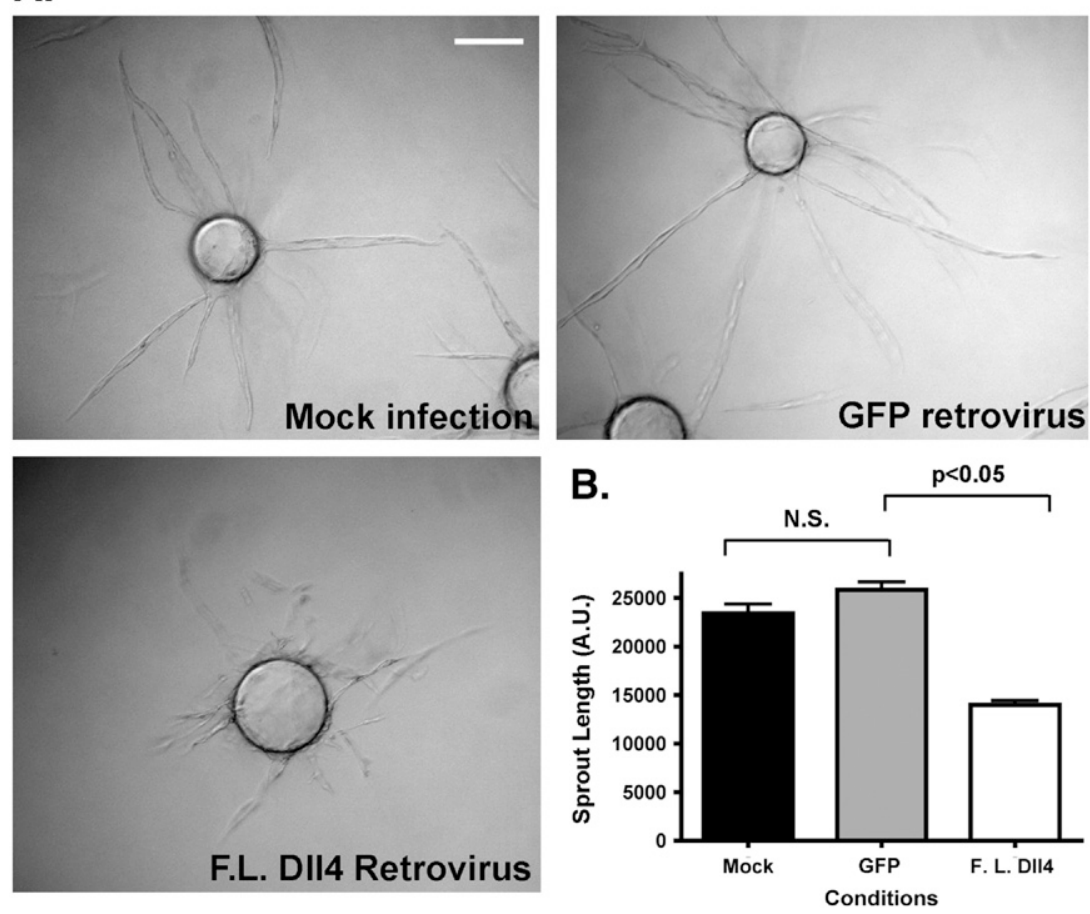

Fig. 4. Ectopic expression of Dll4 inhibits angiogenesis. Single donor HUVECs were infected by retrovirus to express GFP or full-length Dll4 and were compared to mock infected cells for their ability to form tubes in an in vitro 3D-tubulogenesis assay. (A) Phase contrast images show a decrease in vessel length (at day 10) following exogenous expression of Dl14. Scale bar represents $100 \mu \mathrm{m}$. (B) Quantification of vessel length in arbitrary units (a minimum of 100 sprouts was quantified). Results from one experiment representative of three independent experiments are shown.

A.
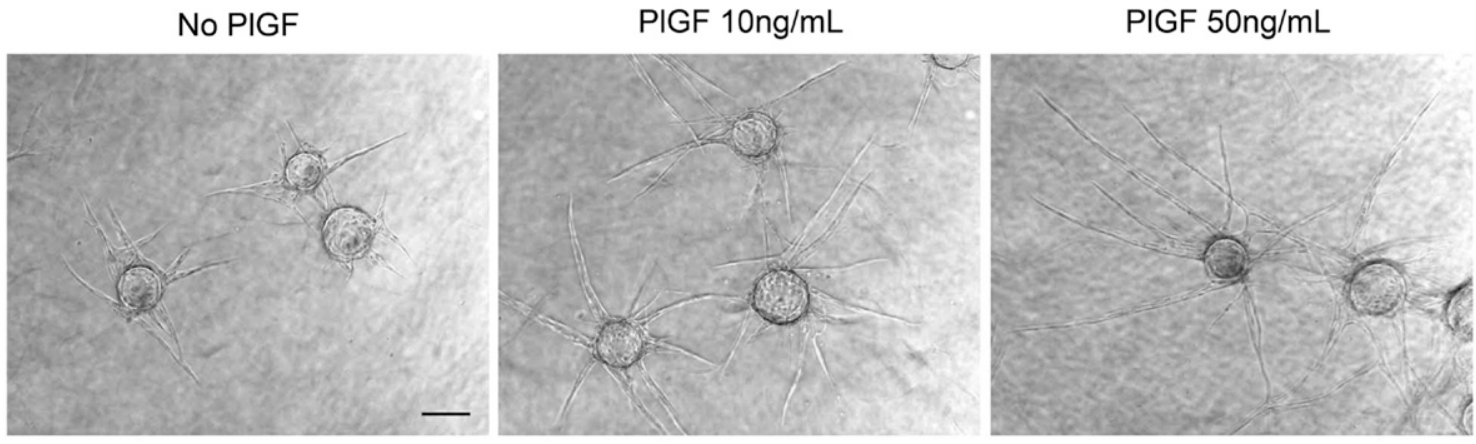

B.

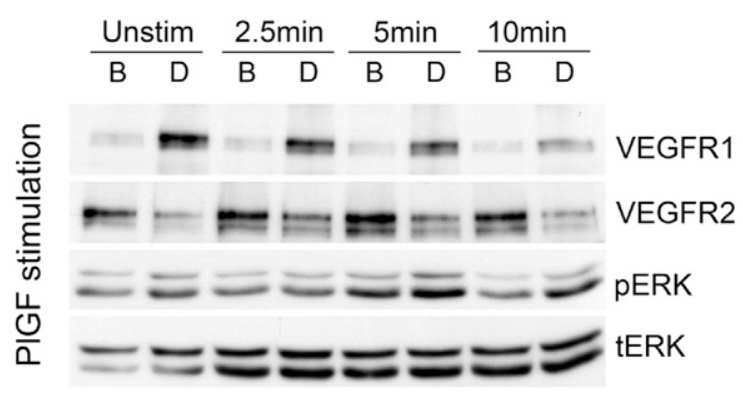

Fig. 5. PlGF enhances tubulogenesis and ERK activation in HUVECs cultured on rDll4-coated plates. (A) HUVECs placed in the 3D tubulogenesis assay were treated 2 days later with vehicle, $10 \mathrm{ng} / \mathrm{mL}$ or $50 \mathrm{ng} / \mathrm{mL}$ PlGF-supplemented media changing the media every other day for 5 treatments. PlGF treatment led to enhanced tube formation. Scale bar, $100 \mu \mathrm{m}$. (B) HUVECs were cultured on BSA- or rDll4-coated plates for $48 \mathrm{~h}$ and starved for $5 \mathrm{~h}$ prior to stimulation with fresh starvation media supplemented with $10 \mathrm{ng} / \mathrm{mL}$ PIGF for the indicated times. PIGF stimulation resulted in enhanced and prolonged ERK activation in HUVECs cultured on rDll4-coated plates compared to control. One experiment representative of three is shown. 
reminiscent of but reciprocal to the role of the orphan nuclear receptor, COUP-TFII, which suppresses Notch signalling and arterial markers such as NRP1 to regulate venous cell fate (You et al., 2005).

Only four genes from our microarray were also regulated by Hey2 overexpression in HUVECs (Chi et al., 2003). The small degree of concordance may reflect differences in array technologies and relative degrees of expression of the constructs but emphasises potential specificity as D114 regulates genes independently of one downstream factor, Hey2.

In conclusion, in order to understand the molecular mechanisms behind the effects of D114 on EC biology, elucidation of the signal transduction pathways regulated specifically by D114 in ECs is essential. Here we report the use of a microarray screen to systematically identify novel Dll4 targets. In accordance with our in vitro 3D tubulogenesis assay and recent in vivo data, we suggest that Dll4 limits EC proliferation and angiogenesis and triggers the maturation and differentiation of vessels possibly via the novel Dll4 targets identified herein. Elucidation of the functions of these targets in angiogenesis is the focus of our ongoing work.

\section{Acknowledgments}

Research in the laboratory are supported by Cancer Research UK; EU 6thFramework Angiotargeting.

\section{Appendix A. Supplementary data}

Supplementary data associated with this article can be found, in the online version, at doi:10.1016/j.mvr.2007.06.006.

\section{References}

Albig, A.R., Neil, J.R., Schiemann, W.P., 2006. Fibulins 3 and 5 antagonize tumor angiogenesis in vivo. Cancer Res. 66, 2621-2629.

Artavanis-Tsakonas, S., Rand, M.D., Lake, R.J., 1999. Notch signaling: cell fate control and signal integration in development. Science 284, 770-776.

Battle, T.E., Lynch, R.A., Frank, D.A., 2006. Signal transducer and activator of transcription 1 activation in endothelial cells is a negative regulator of angiogenesis. Cancer Res. 66, 3649-3657.

Benedito, R., Duarte, A., 2005. Expression of D114 during mouse embryogenesis suggests multiple developmental roles. Gene Expr. Patterns 5, 750-755.

Cai, J., Ahmad, S., Jiang, W.G., Huang, J., Kontos, C.D., Boulton, M., Ahmed, A., 2003. Activation of vascular endothelial growth factor receptor-1 sustains angiogenesis and Bcl-2 expression via the phosphatidylinositol 3-kinase pathway in endothelial cells. Diabetes 52, 2959-2968.

Chi, J.T., Chang, H.Y., Haraldsen, G., Jahnsen, F.L., Troyanskaya, O.G., Chang, D.S., Wang, Z., Rockson, S.G., van de Rijn, M., Botstein, D., Brown, P.O., 2003. Endothelial cell diversity revealed by global expression profiling. Proc. Natl. Acad. Sci. U. S. A. 100, 10623-10628.

Claxton, S., Fruttiger, M., 2004. Periodic Delta-like 4 expression in developing retinal arteries. Gene Expr. Patterns 5, 123-127.

Duarte, A., Hirashima, M., Benedito, R., Trindade, A., Diniz, P., Bekman, E., Costa, L., Henrique, D., Rossant, J., 2004. Dosage-sensitive requirement for mouse D114 in artery development. Genes Dev. 18, 2474-2478.

Ferrara, N., Gerber, H.P., LeCouter, J., 2003. The biology of VEGF and its receptors. Nat. Med. 9, 669-676.

Gale, N.W., Dominguez, M.G., Noguera, I., Pan, L., Hughes, V., Valenzuela, D.M., Murphy, A.J., Adams, N.C., Lin, H.C., Holash, J., et al., 2004. Haploinsufficiency of delta-like 4 ligand results in embryonic lethality due to major defects in arterial and vascular development. Proc. Natl. Acad. Sci. U. S. A. 101, 15949-15954.

Hainaud, P., Contreres, J.O., Villemain, A., Liu, L.X., Plouet, J., Tobelem, G., Dupuy, E., 2006. The role of the vascular endothelial growth factor-deltalike 4 ligand/Notch4-Ephrin B2 cascade in tumor vessel remodeling and endothelial cell functions. Cancer Res. 66, 8501-8510.

Hellstrom, M., Phng, L.K., Hofmann, J.J., Wallgard, E., Coultas, L., Lindblom, P., Alva, J., Nilsson, A.K., Karlsson, L., Gaiano, N., et al., 2007. Dl14 signalling through Notch1 regulates formation of tip cells during angiogenesis. Nature 445, 776-780.

Irizarry, R.A., Hobbs, B., Collin, F., Beazer-Barclay, Y.D., Antonellis, K.J., Scherf, U., Speed, T.P., 2003. Exploration, normalization, and summaries of high density oligonucleotide array probe level data. Biostatistics 4, $249-264$

Iso, T., Hamamori, Y., Kedes, L., 2003a. Notch signaling in vascular development. Arterioscler. Thromb. Vasc. Biol. 23, 543-553.

Iso, T., Kedes, L., Hamamori, Y., 2003b. HES and HERP families: multiple effectors of the Notch signaling pathway. J. Cell. Physiol. 194, 237-255.

Iso, T., Maeno, T., Oike, Y., Yamazaki, M., Doi, H., Arai, M., Kurabayashi, M., 2006. Dl14-selective Notch signaling induces ephrinB2 gene expression in endothelial cells. Biochem. Biophys. Res. Commun. 341, 708-714.

Karsan, A., 2005. The role of notch in modeling and maintaining the vasculature. Can. J. Physiol. Pharmacol. 83, 14-23.

Krebs, L.T., Shutter, J.R., Tanigaki, K., Honjo, T., Stark, K.L., Gridley, T., 2004. Haploinsufficient lethality and formation of arteriovenous malformations in Notch pathway mutants. Genes Dev. 18, 2469-2473.

Lai, E.C., 2004. Notch signaling: control of cell communication and cell fate. Development 131, 965-973.

Liu, Z.J., Shirakawa, T., Li, Y., Soma, A., Oka, M., Dotto, G.P., Fairman, R.M., Velazquez, O.C., Herlyn, M., 2003. Regulation of Notch1 and Dll4 by vascular endothelial growth factor in arterial endothelial cells: implications for modulating arteriogenesis and angiogenesis. Mol. Cell. Biol. 23, 14-25.

Lobov, I.B., Renard, R.A., Papadopoulos, N., Gale, N.W., Thurston, G., Yancopoulos, G.D., Wiegand, S.J., 2007. Delta-like ligand 4 (D114) is induced by VEGF as a negative regulator of angiogenic sprouting. Proc. Natl. Acad. Sci. U. S. A. 104, 3219-3224.

Mailhos, C., Modlich, U., Lewis, J., Harris, A., Bicknell, R., Ish-Horowicz, D., 2001. Delta4, an endothelial specific notch ligand expressed at sites of physiological and tumor angiogenesis. Differentiation 69, 135-144.

Nakatsu, M.N., Sainson, R.C., Aoto, J.N., Taylor, K.L., Aitkenhead, M., Perezdel-Pulgar, S., Carpenter, P.M., Hughes, C.C., 2003. Angiogenic sprouting and capillary lumen formation modeled by human umbilical vein endothelial cells (HUVEC) in fibrin gels: the role of fibroblasts and Angiopoietin-1. Microvasc. Res. 66, 102-112.

Noguera-Troise, I., Daly, C., Papadopoulos, N.J., Coetzee, S., Boland, P., Gale, N.W., Lin, H.C., Yancopoulos, G.D., Thurston, G., 2006. Blockade of Dl14 inhibits tumour growth by promoting non-productive angiogenesis. Nature 444, 1032-1037.

Noseda, M., Chang, L., McLean, G., Grim, J.E., Clurman, B.E., Smith, L.L., Karsan, A., 2004. Notch activation induces endothelial cell cycle arrest and participates in contact inhibition: role of p21Cip1 repression. Mol. Cell. Biol. 24, 8813-8822.

Patel, N.S., Li, J.L., Generali, D., Poulsom, R., Cranston, D.W., Harris, A.L., 2005. Up-regulation of delta-like 4 ligand in human tumor vasculature and the role of basal expression in endothelial cell function. Cancer Res. 65, 8690-8697.

Patel, N.S., Dobbie, M.S., Rochester, M., Steers, G., Poulsom, R., Le Monnier, K., Cranston, D.W., Li, J.L., Harris, A.L., 2006. Up-regulation of endothelial delta-like 4 expression correlates with vessel maturation in bladder cancer. Clin. Cancer Res. 12, 4836-4844.

Ridgway, J., Zhang, G., Wu, Y., Stawicki, S., Liang, W.C., Chanthery, Y., Kowalski, J., Watts, R.J., Callahan, C., Kasman, I., et al., 2006. Inhibition of D114 signalling inhibits tumour growth by deregulating angiogenesis. Nature 444, 1083-1087.

Rosario, M., Birchmeier, W., 2003. How to make tubes: signaling by the Met receptor tyrosine kinase. Trends Cell Biol. 13, 328-335. 
Sainson, R.C., Aoto, J., Nakatsu, M.N., Holderfield, M., Conn, E., Koller, E., Hughes, C.C., 2005. Cell-autonomous notch signaling regulates endothelial cell branching and proliferation during vascular tubulogenesis. FASEB J. 19, 1027-1029.

Scehnet, J.S., Jiang, W., Ram Kumar, S., Krasnoperov, V., Trindade, A., Benedito, R., Djokovic, D., Borges, C., Ley, E.J., Duarte, A., Gill, P.S., 2007. Inhibition of Dll4-mediated signaling induces proliferation of immature vessels and results in poor tissue perfusion. Blood 109, 4753-4760.

Shawber, C.J., Kitajewski, J., 2004. Notch function in the vasculature: insights from zebrafish, mouse and man. BioEssays 26, 225-234.

Shutter, J.R., Scully, S., Fan, W., Richards, W.G., Kitajewski, J., Deblandre, G.A., Kintner, C.R., Stark, K.L., 2000. Dl14, a novel Notch ligand expressed in arterial endothelium. Genes Dev. 14, 1313-1318.

Smyth, G.K., 2004. Linear models and empirical bayes methods for assessing differential expression in microarray experiments. Stat. Appl. Genet. Mol. Biol. 3, 1-26.

Smyth, G.K., Speed, T., 2003. Normalization of cDNA microarray data. Methods 31, 265-273.

Stella, M.C., Trusolino, L., Pennacchietti, S., Comoglio, P.M., 2005. Negative feedback regulation of Met-dependent invasive growth by Notch. Mol. Cell. Biol. 25, 3982-3996.
Suchting, S., Freitas, C., le Noble, F., Benedito, R., Breant, C., Duarte, A., Eichmann, A., 2007. The Notch ligand Delta-like 4 negatively regulates endothelial tip cell formation and vessel branching. Proc. Natl. Acad. Sci. U. S. A. 104, 3225-3230.

Varnum-Finney, B., Wu, L., Yu, M., Brashem-Stein, C., Staats, S., Flowers, D., Griffin, J.D., Bernstein, I.D., 2000. Immobilization of Notch ligand, Delta-1, is required for induction of notch signaling. J. Cell Sci. 113 (Pt. 23), 4313-4318.

Villa, N., Walker, L., Lindsell, C.E., Gasson, J., Iruela-Arispe, M.L., Weinmaster, G., 2001. Vascular expression of Notch pathway receptors and ligands is restricted to arterial vessels. Mech. Dev. 108, 161-164.

Williams, C.K., Li, J.L., Murga, M., Harris, A.L., Tosato, G., 2006. Upregulation of the Notch ligand Delta-like 4 inhibits VEGF-induced endothelial cell function. Blood 107, 931-939.

Yang, B., Cao, D.J., Sainz, I., Colman, R.W., Guo, Y.L., 2004. Different roles of ERK and p38 MAP kinases during tube formation from endothelial cells cultured in 3-dimensional collagen matrices. J. Cell. Physiol. 200, 360-369.

You, L.R., Lin, F.J., Lee, C.T., DeMayo, F.J., Tsai, M.J., Tsai, S.Y., 2005. Suppression of Notch signalling by the COUP-TFII transcription factor regulates vein identity. Nature 435, 98-104. 